\title{
BINARY BLACK HOLES COALESCENCE: TRANSITION FROM ADIABATIC INSPIRAL TO PLUNGE *
}

\author{
ALESSANDRA BUONANNO \\ Theoretical Astrophysics and Relativity Group \\ California Institute of Technology, Pasadena, CA, 91125, USA \\ E-mail: buonanno@tapir.caltech.edu \\ THIBAULT DAMOUR \\ Institut des Hautes Etudes Scientifiques, 91440 Bures-sur-Yvette, France \\ E-mail: damour@ihes.fr

\begin{abstract}
Using two recent techniques giving non-perturbative re-summed estimates of the damping and of the conservative part of the dynamics of two-body systems, we describe the transition between adiabatic inspiral and plunge in binary non-spinning black holes moving along quasi-circular orbits.
\end{abstract}

It seems likely that the first detection of gravitational waves with the LIGO/VIRGO/... interferometers will come from binary systems made of massive black holes of comparable masses, say with a total mass $M \simeq 15 M_{\odot}+15 M_{\odot}$. For such massive systems the gravitational-wave frequency at the last stable (circular) orbit (LSO) is very close to the location of the minimum of the detector's noise spectral density. Therefore, for data analysis purposes it is quite desirable to have a thorough knowledge of the late dynamical evolution of comparable-mass binaries.

When the two black holes become closer than $\sim 10 M$, the post-Newtonian $(\mathrm{PN})$ expansion, which adequately describes the motion at large separations, cannot be trusted any more, and non-perturbative techniques should be used to deal with the non linearities and the strong curvature effects of the final phase of evolution. Despite the progress made by the numerical relativity community during the last years, an estimate of the complete waveform emitted by a comparable-mass black hole binary has not been provided, yet. Hence, to tackle this issue we have recently introduced a new non-perturbative analytical approach to the general relativistic two-body problem 1 , 2 which should be able to capture the crucial features of the transition from the adiabatic phase to the plunge.

Our approach combines two PN re-summation techniques. The first method 1 allows one to derive a non-perturbative estimate of the conservative part of the nonlinear force law determining the motion of comparable-mass binaries. The basic idea $\mathrm{U}$ is to map the conservative real two-body dynamics (up to $2 \mathrm{PN}$ order) onto an effective one-body one, where a test particle of mass $\mu \equiv m_{1} m_{2} / M$ moves in some effective background metric $g_{\mu \nu}^{\text {eff }}$. As long as radiation reaction effects are not taken into account, the effective metric is just a deformation of the Schwarzschild metric with deformation parameter $\nu=\mu / M$. The effective description provides a way of re-summing in a non-perturbative manner the badly convergent $\mathrm{PN}$-expanded

*CONTRIBUTED PAPER TO THE IX MARCEL GROSSMANN MEETING IN ROME, JULY 2000 .

talk: submitted to World Scientific on October 22, 2018 
dynamics of the real description, giving the following improved real Hamiltonian 6 :

$$
H_{\text {real }}^{\text {improved }}=M c^{2} \sqrt{1+2 \nu\left(\frac{H_{\mathrm{eff}}^{\nu}-\mu c^{2}}{\mu c^{2}}\right)} .
$$

The second non-perturbative technique $\mathrm{B}$ concerns radiation reaction effects, and gives a re-summed estimate (using Padé approximants) of the energy loss rate along circular orbits, $\Phi_{\text {circ }}$ (up to $2.5 \mathrm{PN}$ order). Combining the two re-summation methods yields a system of ordinary differential equations, which in spherical coordinates $\left(\varphi, R, P_{\varphi}, P_{R}\right)$ reads 2 :

$$
\frac{d R}{d t}=\frac{\partial H_{\mathrm{real}}^{\mathrm{impr}}}{\partial P_{R}}, \quad \frac{d P_{R}}{d t}+\frac{\partial H_{\mathrm{real}}^{\mathrm{impr}}}{\partial R}=0, \quad \frac{d \varphi}{d t}=\frac{\partial H_{\mathrm{real}}^{\mathrm{impr}}}{\partial P_{\varphi}}, \quad \frac{d P_{\varphi}}{d t}=-\frac{\Phi_{\mathrm{circ}}}{\dot{\varphi}} .
$$

When working linearly in the radial velocity $\dot{R}$, the following characteristic equation describing the transition from the adiabatic inspiral to the plunge was derived 6 :

$$
\frac{d^{3} R}{d t^{3}}+\omega_{R}^{2}(R) \frac{d R}{d t}=-B_{R}(R) .
$$

The quantity $\omega_{R}^{2}$ plays the role of a "restoring force". It is the square of the frequency of radial oscillations and is proportional to the curvature of the effective radial potential (it vanishes at the LSO). The quantity $-B_{R}(\propto \nu)$ is a "driving force", coming from gravitational radiation damping. The term $d^{3} R / d t^{3}$ is an "inertia term", which is neglected in the adiabatic approximation, but should be retained when describing the motion in proximity of the LSO and beyond it. An equation analogous to Eq. (3) has also been independently derived recently 6 for a test particle moving along quasi-circular equatorial orbits in Kerr spacetime. Our approach has been recently extended to the $3 \mathrm{PN}$ level $\mathrm{E}$.

Using Eg. (2) and the canonical mapping between the effective and the real description 4 , we can: (i) provide initial dynamical data, for numerical relativity investigations, for black holes that have just started their plunge motion and (ii) give, for data analysis purposes, an estimate of the gravitatifnal waveform emitted throughout the inspiral, plunge and ring-down phases (see $\mathrm{d}$ for the data analysis consequences of this waveform).

Let us finally mention two interesting facts: (1) the energy emitted during the plunge phase is only around $0.7 \% M$, with a comparable energy loss $\sim 0.7 \% M$ during the following ring-down phase, and (2) the phasing of the gravitational wave signal is essentially unchanged if one turns off the radiation reaction term in Eq. (2), after LSO crossing.

\section{References}

1. A. Buonanno and T. Damour, Phys. Rev. D 59, 084006 (1999).

2. A. Buonanno and T. Damour, Phys. Rev. D 62, 064015 (2000).

3. T. Damour, B.R. Iyer and B.S. Sathyaprakash, Phys. Rev. D 57, 885 (1998).

4. A. Ori and K.S. Thorne, [gr-qc/003032].

5. T. Damour, P. Jaranowski and G. Schäfer, Phys. Rev. D 62, 084011 (2000).

6. T. Damour, B.R. Iyer and B.S. Sathyaprakash, gr-qc/0010009.

talk: submitted to World Scientific on October 22, 2018 